\section{Use of the multi-cup denture for a severely resorbed maxilla: a clinical report}

\author{
R. Vasant ${ }^{1}$ and G. S. Bassi ${ }^{2}$
}

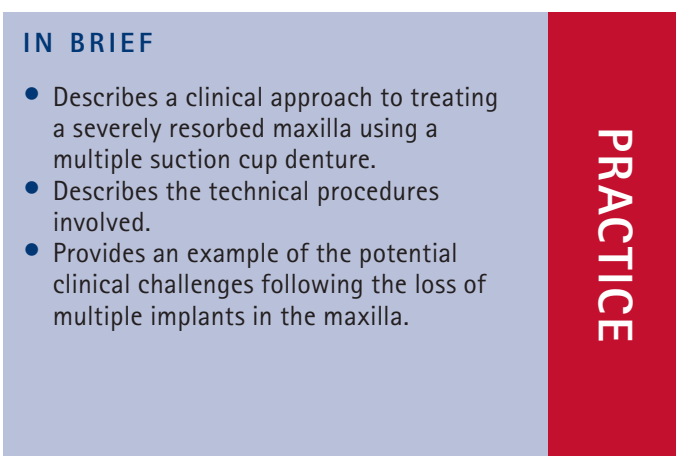

Obtaining a satisfactory outcome for a patient with a severely resorbed maxilla can be extremely challenging. This clinical report describes the treatment of a patient with a history of multiple implant failures in the maxilla. A conventional upper complete denture had poor retention and so did not fulfil the patient's needs. Further surgery was not considered appropriate. A new upper complete denture was made with a fitting surface covered in multiple suction cups to attempt to improve the retention without surgery and resulted in a pleasing outcome for both the patient and authors.

\section{INTRODUCTION}

The treatment of a patient with a severely resorbed maxilla can be greatly testing. There can be difficulties in achieving adequate retention and stability with conventional prostheses and whilst dental implants can be used in some situations and be of great benefit, they are not always appropriate. In these situations, alternative methods may have to be considered.

This clinical report explains the successful use of a multi-cup denture to treat a patient who presented with a severely resorbed edentulous maxilla. The technique depicted in this paper has been adapted from that originally described by Jermyn in $1967 .{ }^{1}$ The technique has been recently revived for specific cases where implant treatment was either inappropriate or had already failed. ${ }^{2}$

\section{CASE REPORT}

The authors were presented with a 46-yearold female patient with a history of multiple failed attempts at implant treatment in her maxilla. This had involved two sets of implants being placed at different times, with additional bone grafting from the

\footnotetext{
"Senior Clinical Teaching Fellow, UCL Eastman CPD, 123 Gray's Inn Road, London; ${ }^{2}$ Consultant in Restorative Dentistry, Eastman Dental Hospital, 256 Gray's Inn Road, London

${ }^{*}$ Correspondence to: Mr Ronuk Vasant

Email:ronuk_vasant@hotmail.com
}

\section{Refereed Paper}

Accepted 11 January 2012

DOI: 10.1038/sj.bdj.2012.369

${ }^{\circledR}$ British Dental Journal 2012; 212: 431-434 iliac crest and calvarium. Furthermore, a soft tissue graft had been taken from the inner labial mucosa of the upper lip and 14 implants had been removed, with the remaining ones not fully integrated and in unfavourable positions for restoration. The patient was left with a severely resorbed maxilla (Fig. 1). It can be seen that denture stomatitis was present. In addition there was significant alteration of the sulcus anatomy with an almost complete lack of sulcus and the alveolar ridge was severely resorbed and uneven in contour. The lower arch, however, was reasonably well aligned and partially dentate. There were two implant retained crowns replacing the lower left premolars and the remaining natural teeth were moderately restored. There was evidence of tooth surface loss on the incisal edges of the lower anteriors. The occlusal vertical dimension appeared to be satisfactory. The incisors were in a Class I relationship, with group function on right and left lateral excursion.

An attempt at a conventional upper complete denture was not successful due to poor retention and stability. Further surgery, including supplementary grafting and implant placement, was considered but ultimately rejected due to the high risk of failure. Therefore, it was decided to attempt the use of a multiple suction cup denture to improve retention and stability; though the same materials as those described by Jermyn ${ }^{1}$ could not be used due to the lack of a European distributor and CE Mark. A heat-cured silicone soft reline material, Molloplast B (Detax GmBH,

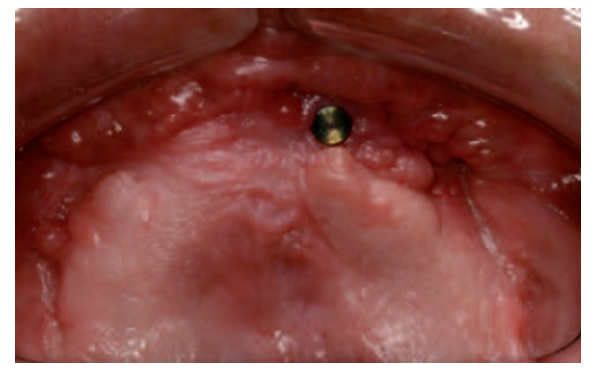

Fig. 1 Occlusal view of maxilla

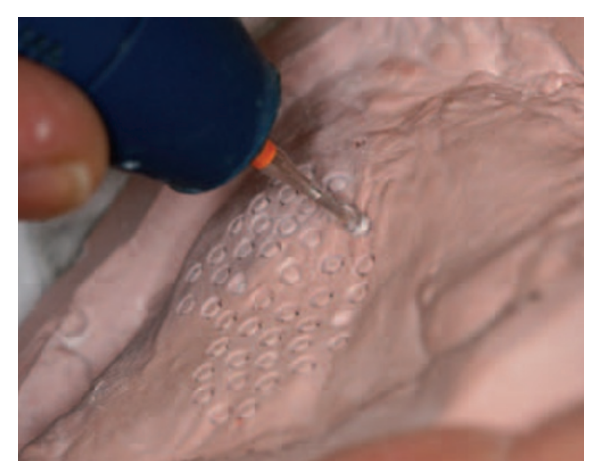

Fig. 2 Drilling of trephine holes into master cast

Ettlingen, Germany), was used instead. This was based on the authors' and technician's familiarity with the material as well as its long history of successful clinical use.

Following the conventional prosthodontic stages of primary impressions, secondary impressions and jaw registration, a tooth try-in on a cold-cure acrylic resin primary base was carried out. Once patient and clinician were happy with the tooth arrangement, the try-in was disinfected and returned to the laboratory. The master cast was then prepared by drilling trephine holes perpendicularly into it (Fig. 2). These 
were placed approximately $1.5 \mathrm{~mm}$ apart, were $1 \mathrm{~mm}$ deep and had a diameter of $2 \mathrm{~mm}$. The entire alveolar ridge and palatal denture supporting areas were prepared in this way, leaving $2 \mathrm{~mm}$ at the denture borders and frenal attachment sites.

The primary base was then sealed down on the master cast with wax in the conventional way (Fig. 3). The denture was then flasked and the wax boiled out in the normal manner (Figs 4 and 5).

At this stage, a new $1.5 \mathrm{~mm}$ thick wax spacer was placed over the entire fitting surface of the cast (Fig. 6). This prevented the acrylic resin dough from entering the trephine holes and allowed sufficient space for the addition of Molloplast B at a later stage.

Heat-cured acrylic resin dough was packed over the teeth in the conventional way and covered with a thin plastic sheet (Fig. 7). After one hour in the press at two bar pressure, the flask was opened. Molloplast B adhesive was applied to the surface of the partially cured acrylic resin, then pressed over the surface of the master cast (Figs 8 and 9).

The flask was closed together and the acrylic allowed to fully cure using standard laboratory protocols. The flask was opened carefully, taking care not to tear the Molloplast B (Fig. 10). The acrylic resin was finished and polished in the conventional manner, again taking care not to damage the Molloplast B (Fig. 11).

The denture was then fitted (Fig. 12). The patient found it very comfortable to wear and reported increased retention compared to her previous conventional prosthesis. This is in line with a previously published report. ${ }^{2}$ The patient was to be followed up for any deleterious soft tissue changes. However, in the previously published case report, ${ }^{2}$ no soft tissue changes were recorded. The patient did not seek any further treatment 12 months after the maxillary denture was fitted.

\section{DISCUSSION}

This case describes the management of a patient who presented with multiple failed implants. The reported success rates of dental implant treatments have been high. The classical studies report 15 year implant survival rates of $86 \%$ in the maxilla, and 99\% in the mandible. ${ }^{3}$ These studies reported on implants placed

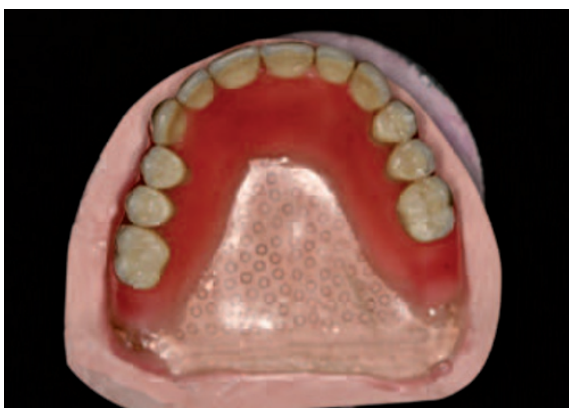

Fig. 3 Sealed down primary base

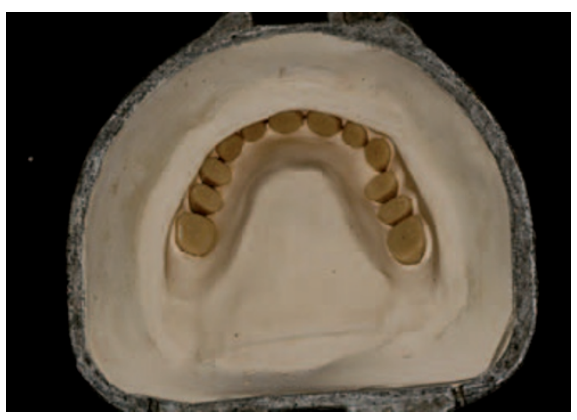

Fig. 4 Acrylic teeth in opposing half of flask following wax elimination

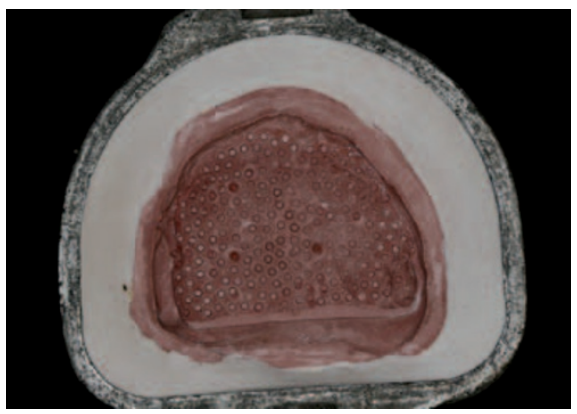

Fig. 5 Master cast following the boiling out procedure

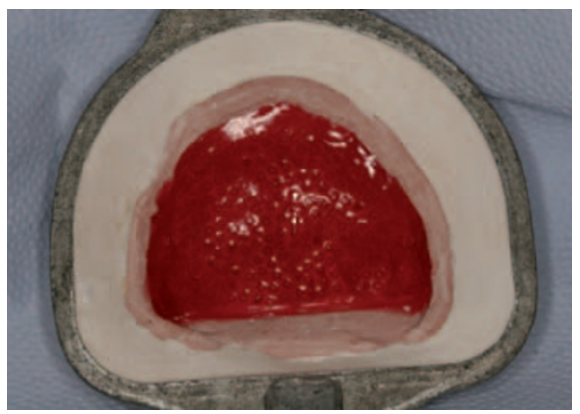

Fig. 6 Application of wax spacer

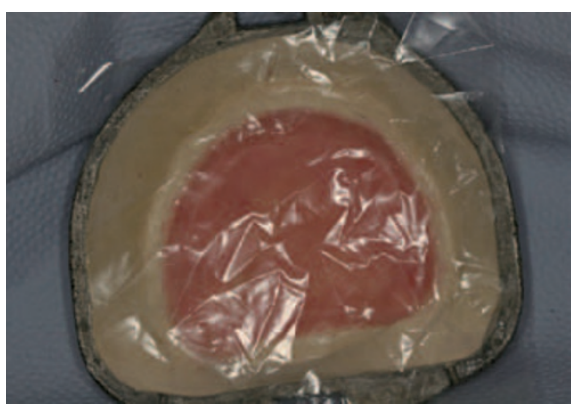

Fig. 7 Acrylic resin dough packed over the teeth

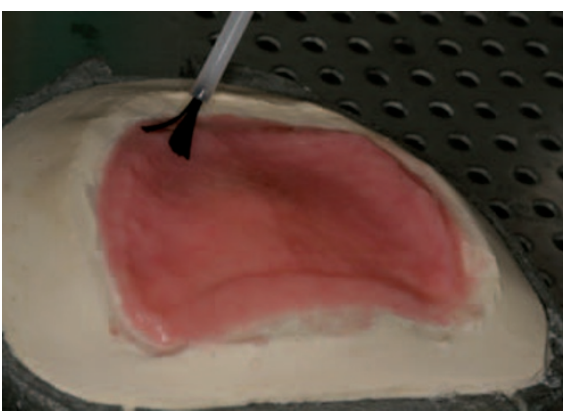

Fig. 8 Adhesive being applied

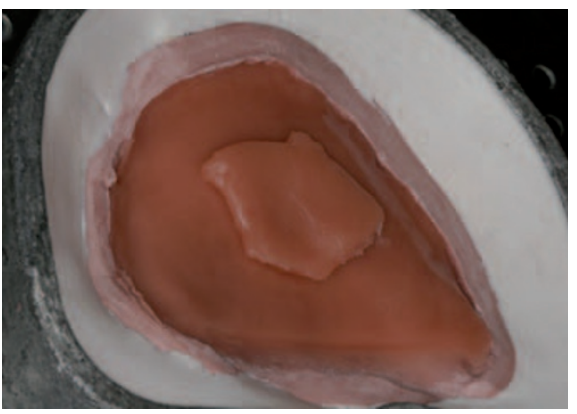

Fig. 9 Molloplast B being placed over the trephine holes on the master cast

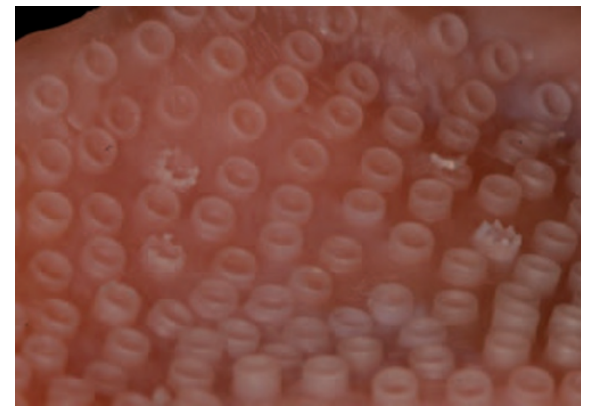

Fig. 10 Close-up of intaglio surface showing tiny suction cups

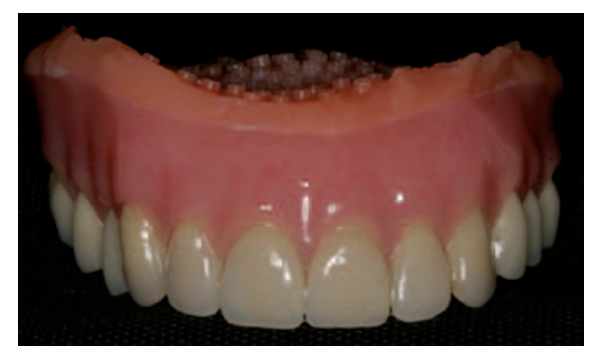

Fig. 11 Finished denture

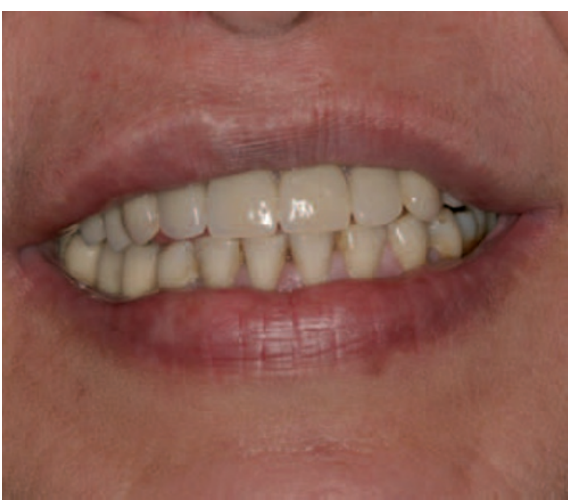

Fig. 12 Patient wearing finished denture 
into non-grafted sites. The patient in this report underwent two separate bone grafting procedures, where autogenous bone was harvested from extra-oral sites. On one occasion the iliac crest was chosen as the donor site, on the other occasion the calvarium was chosen. Adell et al. in $1990^{4}$ reported the survival rates of implants placed into grafted iliac bone in the maxilla. At five years, the survival rate was 73.8\%. It could therefore be expected that there would be an increased risk of implant failure for fixtures placed into grafted iliac bone in the maxilla. In the presented case, the patient had experienced failure of all the implants placed into her maxilla, including the loss of 14 . Unfortunately, despite attempts to contact the previous dentist, access to any clinical records regarding her previous treatment was not gained. Therefore, the reasons why this particular patient suffered such a large number of implant failures in her maxilla can only be speculated upon. There may be several important factors and each will be discussed in turn.

It is assumed that the implants were made of commercially pure titanium. However, in this case, the implant placement was done in Brazil and information regarding the type of implants used is unknown. There are known to be hundreds of small companies across the world producing low-cost implants. Many of these are copies of popular implant designs from well-known manufacturers. It is fairly common for these to be sold without any supporting clinical evidence for their ability to osseointegrate. It is possible therefore, that the implants were contaminated titanium, or indeed not titanium at all.

Poor surgical technique can result in heating the bone by more than $4^{\circ} \mathrm{C}$ during osteotomy preparation and this temperature rise is known to cause damage to osteocytes. ${ }^{4}$ This can result in failure of osseointegration, which may have happened in this case. Normally, the use of saline as a coolant is recommended during the drilling phases of osteotomy preparation.

The patient reported a history of cigarette smoking, but claims that she did not smoke after the implant surgeries. However, cigarette smoking has been shown to be a risk to implant success. ${ }^{5}$
There is some evidence that the presence of micromotion during the healing phase may have detrimental effects on success rates. ${ }^{6-8}$ If complete denture use is necessary following maxillary implant placement, it is conventional to either relieve the denture fit surface over the implants either with or without the addition of a soft reline material. The authors have no details as to how this particular case was managed in that respect.

It is likely that the major reason for the severe loss of alveolar height and width in this case was the history of multiple failed implants that were removed. Periimplantitis results in the loss of bone immediately surrounding implants and is a relatively common complication. Fransson reported that out of 662 patients who had received implant treatment, progressive bone loss was identified in 184 (27.8\%). ${ }^{9}$ Many older studies do not provide adequate data on the prevalence of peri-implantitis. Hutton et al. demonstrated that in a population treated with implant overdentures, many implant failures seemed to cluster in specific individuals, rather then being evenly distributed throughout the studied population. ${ }^{10}$ This case represents such an example of cluster failure.

The use of a suction cup to aid retention is a well-known physical principle. The flexible suction cup is applied to a surface, forcing the air inside the suction cup to be expelled. When the user stops applying a force to the suction cup the elastic properties of the material cause it to return to its original shape. This increases the volume, lowering the pressure inside the suction cup, compared to atmospheric pressure. It is this pressure differential which creates the retention.

The use of a single suction cup for maxillary denture retention is a well-known, although now derided, technique. There have been numerous case reports reporting mucosal ulceration or palatal perforation caused by the use of single suction cups in maxillary dentures. ${ }^{11-13}$ However, all of these published case reports are of the single suction cup type, not the multiple type as used in this case report. The authors are unaware of any published reports of pathological tissue changes as a result of the use of a multiple suction cup denture.

The long term performance of Molloplast B has been documented in the literature. ${ }^{14,15}$ A six-year retrospective follow-up study on complete dentures with Molloplast B linings showed that common problems were fracture, bleach deterioration, liner separation and yeast deterioration. ${ }^{14}$ Some authors ${ }^{13}$ recommend that bleach be used to disinfect the denture every night in order to reduce the microorganism count. A laboratory study ${ }^{16}$ has shown sodium hypochlorite to be the most effective disinfectant, being more effective than either microwave radiation or leaving the denture dry overnight. There is a conflict between the needs for effective disinfection and maintaining the properties of the materials. It would appear likely that the Mollplast B would need to be replaced on a 3-5 year cycle on average.

In general, there are specific challenges associated with making a complete upper denture opposing a dentate or partially dentate mandibular arch. The occlusal plane of the remaining teeth is often uneven. Therefore, a balanced occlusion is often very difficult or impossible to achieve. This results in occlusal relationships that tend to displace, rather than stabilise, the maxillary denture during function. The set-up of the denture teeth can often be compromised with regards to aesthetics as tooth position is dictated by the remaining mandibular teeth. Kelly ${ }^{17}$ attributed several features to this prosthetic configuration: a loss of bone in the anterior maxillary alveolar ridge region, overgrowth of the tuberosities, papillary hyperplasia of the hard palate, extrusion of the lower anterior teeth and resorption of the posterior mandibular ridge.

\section{SUMMARY}

Dental implant use has increased worldwide over a number of years. They can be an extremely effective method of replacing teeth or stabilising removable prostheses. However, failure in the short- or long-term can still occur despite advances in implant surface technology. Patients who have experienced implant failure may not wish to undergo further surgery, particularly if bone augmentation is necessary and especially if repeated surgery has already been attempted. There are also patients who wish to avoid the surgery associated with implant treatment in the first place or for whom surgery is not advised due to a medical complication. Therefore, a clinician 
should have at his/her disposal a variety of techniques that can be used when implants are not indicated. This article aims to add to the clinician's armamentarium when a conventional maxillary complete denture is unlikely to provide satisfactory retention and stability.

The authors wish to thank Mr Colin Clark, Technical Instructor, Restorative Division, Eastman Dental Institute, for his assistance with the technical aspects of the described case.

1. Jermyn A C. Multiple suction cup dentures. J Prosthet Dent 1967; 18: 316-325.

2. Engelmeier R L, Gonzalez M L, Harb M. Restoration of the severely compromised maxilla using the multi-cup denture. J Prosthodont 2008; 17: 41-46.

3. Adell, R, Eriksson B, Lekholm U, Brånemark, PI, Jemt T. A long-term follow-up study of osseointegrated implants in the treatment of totally edentulous jaws. Int J Oral Maxillofac Implants 1990; 5: 347-359.
4. Eriksson R A, Adell R. Temperatures during drilling for the placement of implants using the osseointegration technique. Int J Oral Maxillofac Surg 1986; 44: 4-7.

5. Bain C A, Moy P K. The association between the failure of dental implants and cigarette smoking. Int J Oral Maxillofac Implants 1993: 8: 609-615.

6. Balshi T J, Wolfinger G J. Immediate loading of Brånemark implants in edentulous mandibles: a preliminary report. Implant Dent 1997; 6: 83-88.

7. Schnitman PA, Wöhrle PS, Rubenstein J E, DaSilva $J$ D, Wang N H. Ten-year results for Brånemark implants immediately loaded with fixed prostheses at implant placement. Int J Oral Maxillofac Implants 1997: 12: 495-503.

8. Tarnow D P, Emtiaz S, Classi A. Immediate loading of threaded implants at stage 1 surgery in edentulous arches: ten consecutive case reports with 1- to 5-year data. Int J Oral Maxillofac Implants 1997; 12: 319-324.

9. Fransson $\mathrm{C}$, Lekholm U, Jemt $\mathrm{T}$, Berglundh $\mathrm{T}$. Prevalence of subjects with progressive loss at implants. Clin Oral Implants Res 2005; 16: 440-446.

10. Hutton J $E_{1}$ Heath M R, Chai J $Y$ et al. Factors related to success and failure rates at 3-year follow-up in a multicenter study of overdentures supported by Brånemark implants. J Oral Maxillofac Implants 1995; 10: 33-42.

11. Ordulu M, Emes Y, Ates M, Aktas I, Yalçin S. Oronasal communication caused by a denture with suction cups: a case report. Quintessence Int 2006; 37: 659-662.

12. Cayetano O, Boone M E. Suction cups on maxillary dentures: report of case. J Am Dent Assoc 1987; 115: 577.

13. Mahabob M N, Sambandan T, Kumar B S. Suction cup induced oronasal communication. JIADS 2010; 1: 43-46.

14. Schmidt W F Jr, Smith D E. A six-year retrospective study of Molloplast-B-lined dentures. Part II: liner serviceability. J Prosthet Dent 1983; 50: 459-465.

15. Ryan J E, Twenty-five years of clinical application of a heat-cured silicone rubber. J Prosthet Dent 1991; 65: 658-660.

16. Baysan A, Whiley $R$, Wright $P S$, Use of microwave energy to disinfect a long-term soft lining material contaminated with Candida albicans or Staphylococcus aureus. J Prosthet Dent 1998; 79: 454-458.

17. Kelly E. Changes caused by a mandibular removable partial denture opposing a maxillary complete denture. J Prosthet Dent 1972; 27: 140-150. 